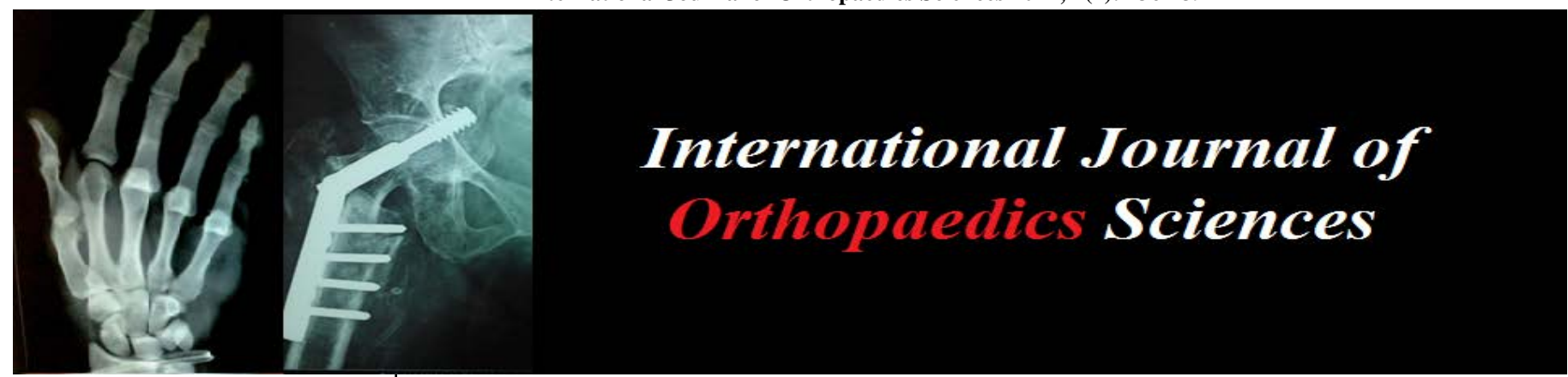

E-ISSN: 2395-1958

P-ISSN: 2706-6630

IJOS 2021; 7(4): 236-239

(C) 2021 IJOS

www.orthopaper.com

Received: 13-07-2021

Accepted: 22-08-2021

Dr. Brajesh Dadarya

Ex Associate Professor,

Department of Orthopaedics

Nscb Medical College, Jabalpur,

Madhya Pradesh, India

Dr. Ajay Dhanopeya

Senior Resident, Department of

Orthopaedics, Fortis Escorts

Heart Institute, New Delhi,

India

Dr. Shamik Muneer Gaus UI

Raza, Assistant Professor,

Department of Orthopaedics

Nscb Jabalpur, Madhya

Pradesh, India

Dr. Mayank Pratap Singh

Senior Resident, Department of

Orthopaedics, Govt. Medical

College, Shivpuri, Madhya

Pradesh, India
Corresponding Author:

Dr. Mayank Pratap Singh Senior Resident, Department of Orthopaedics, Govt. Medical College, Shivpuri, Madhya

Pradesh, India

\section{To study the outcome and efficacy of proximal fibular osteotomy in osteoarthritis knee: Extended follow up study author}

\author{
Dr. Brajesh Dadarya, Dr. Ajay Dhanopeya, Dr. Shamik Muneer Gaus UI \\ and Dr. Mayank Pratap Singh
}

DOI: https://doi.org/10.22271/ortho.2021.v7.i4d.2888

\begin{abstract}
The aim was to study the long term effect of PFO on the predominant medial compartment osteoarthritis knee. In initial period of one year, 40 patients with predominant medial compartment osteoarthritis were operated in department of orthopaedics and followed up for three years. In our study, the average age of presentation was 64.1 year. The outcome was evaluated radiologically by using FTA, JCA and clinically by AKSS score and VAS score. Follow up was done according to set proforma. At final follow up, mean FTA improved to $181.8 \pm 1.159$ degree from $184.05 \pm 1.694$ degree preoperatively and JCA improved to $3.13^{ \pm} 1.223$ degree from $1.3^{ \pm} 0.853$ degree preoperatively (both values were significant $\mathrm{P}<0.001$ ). Mean AKSS improved to $85.95 \pm 12.989$ at final follow up from $33.55 \pm 1.085$ preoperatively and VAS score improved to $1.87 \pm 1.261$ from $8.55^{ \pm} 0.02$ preoperatively (both values were significant $\mathrm{P}<0.001$ ). It was concluded that PFO is a new and cost effective surgery for predominant medial compartment osteoarthritis of knee with better functional outcome of patients and significant decrease in pain as compared to the conventional treatment modalities.
\end{abstract}

Keywords: PFO - Proximal Fibular Osteotomy, FTA - Femorotibial Angle, JCA - Joint Convergence Angle, AKSS - American Knee Society score, VAS - Visual Analogue Scale score, BMI - Body Mass Index

\section{Introduction}

Osteoarthritis is the second most common rheumatologic problem and it is the most frequent joint disease with a prevalence of $22 \%$ to $39 \%$ in India ${ }^{[1,2]}$. OA is more common in women than men, but the prevalence increases dramatically with age ${ }^{[3]}$.

Zhang et al. reported that Proximal Fibular Osteotomy (PFO) relieves pain and improves joint function in knee osteoarthritis ${ }^{[4]}$. This new surgery is simple, safe and affordable.

\section{Methodology}

- The aim of the study was to study the Outcome and Efficacy of Proximal Fibular Osteotomy in Osteoarthritis Knee on the basis of clinical and radiological parameters which was carried out in the Department of Orthopaedics, Traumatology and Rehabilitation N.S.C.B. Medical College Jabalpur (M.P.) and the patients were of 60-69 years age group including both male and female gender. Ethical clearance was obtained before beginning of the study from ethical clearance committee.

- Total 40 knees with medial compartment osteoarthritis were operated during this period. All these patients were included in our study and were followed up prospectively starting from immediate postoperative period, at 3 weeks, 6 weeks, 3 months, 6 months and 1 year. After that all patients were followed up for total three years and various parameters were assessed and noted as per the set proforma.

\section{Inclusion criteria}

1. Patients with moderate to severe symptomatic degenerative osteoarthritis of the knee.

2. Patients on whom conservative management has failed.

3. Patients who have given informed consent. 


\section{Exclusion criteria}

1. Patients who have inflammatory or post traumatic osteoarthritis of knee.

2. Patients who have any history of previous operations or fractures around the knee.

3. Patients who have not given informed consent

\section{Aims \& objective}

1. To study the effects of proximal fibular osteotomy on symptoms specially pain relief of osteoarthritis knee using Visual Analogue Scale (VAS) in long term.

2. To study the functional outcome of proximal fibular osteotomy using American Knee Society Score.

3. To study the changes in joint space using joint convergence angle.

4. To study the outcome measures of pre and post-operative Femorotibial angle using scanogram.

5. Effects of factor like BMI, physiotherapy on long term follow up.

\section{Surgical technique}

We used the Henry's posterolateral approach for exposure of proximal fibula.

- The head of fibula was palpated and surface marking of the head with length of incision and osteotomy site was done.

- Approximately a $5 \mathrm{~cm}$ long incision was made over the posterolateral border of the fibula centred over the point 7 $\mathrm{cm}$ below the head of fibula.

- Fascia between peroneus longus and soleus muscle was identified and cut.

- Fibula was exposed from the posterolateral aspect.

- Again the level of osteotomy was confirmed and $2 \mathrm{~cm}$ of segment of fibula was marked.

- A $2 \mathrm{~cm}$ section of fibula was excised $7 \mathrm{~cm}$ below the fibular head.

- The osteotomy was done by either multiple drill holes and osteotome or Gigli saw or oscillating saw (in this only lateral cortex is cut with oscillating saw; the medial cortex is cut by osteotome).

- The muscles fascia, skin were closed separately in layers after ample irrigation with normal saline.

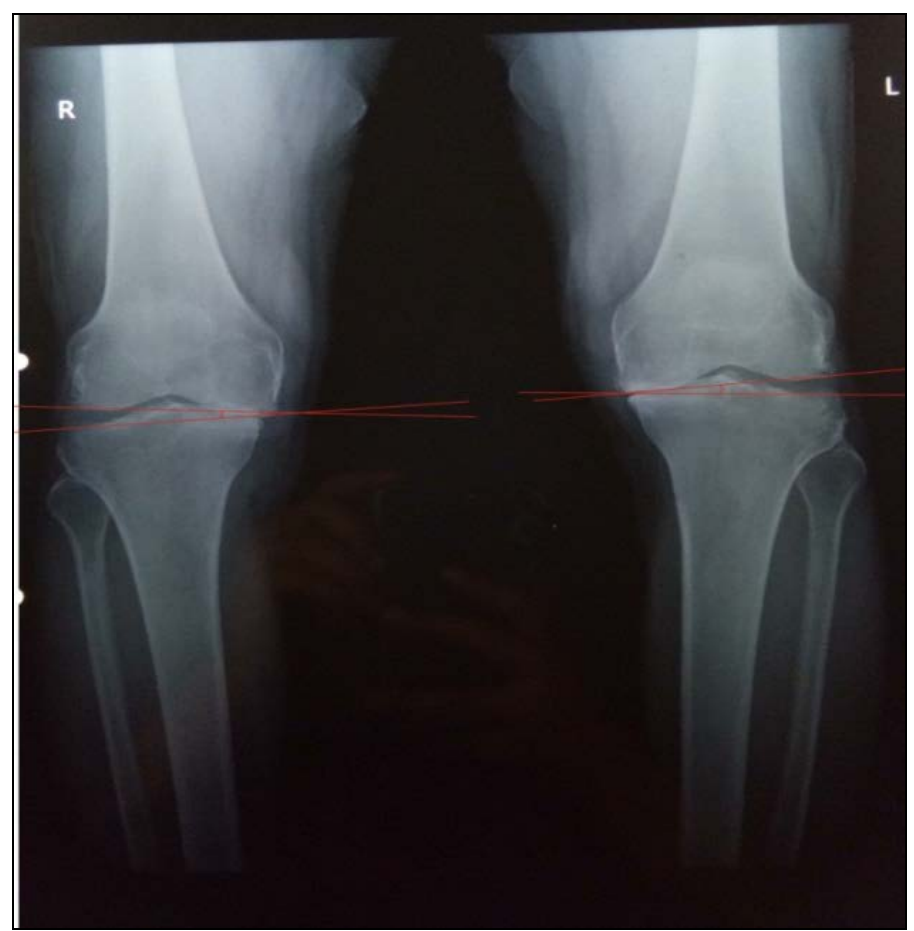

Joint Convergence Angle

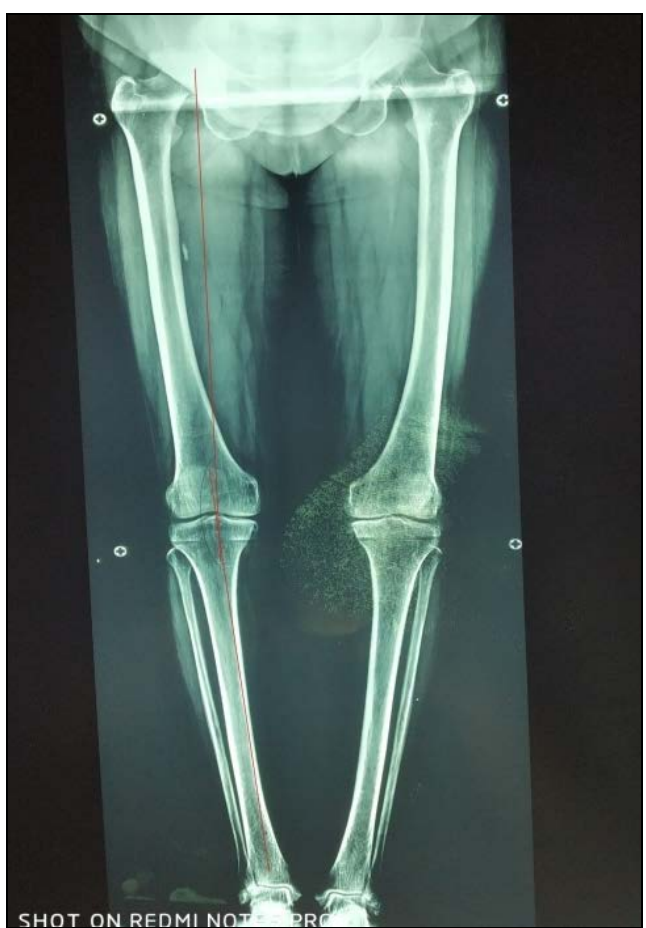

Femorotibial Angle

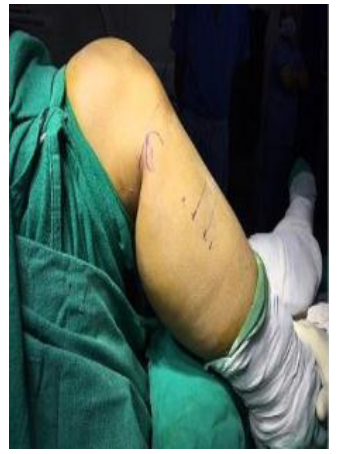

Surface marking of head of fibula with length of incision and osteotomy site

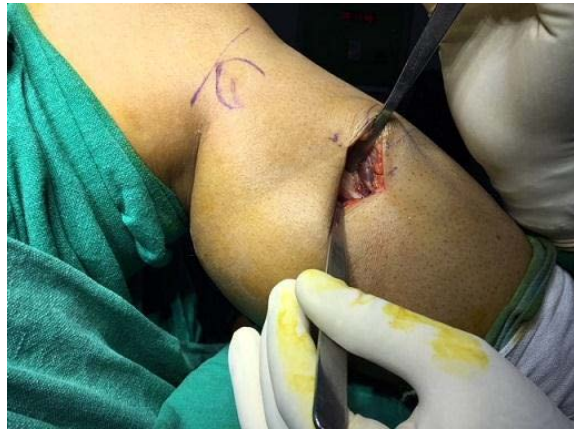

Fibula exposed through posterolateral aspect

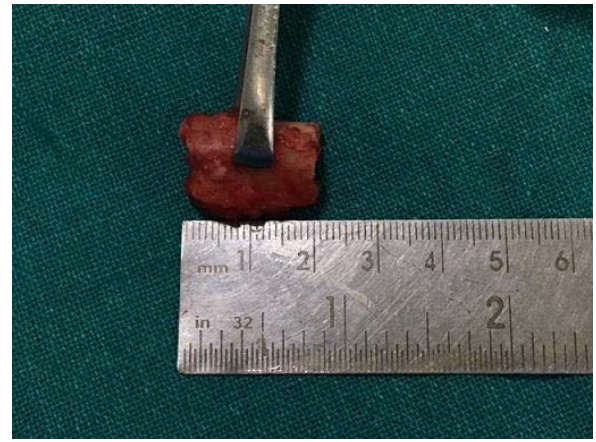

$2 \mathrm{~cm}$ section of fibula 


\section{Pre-Operative \\ Radiograph}

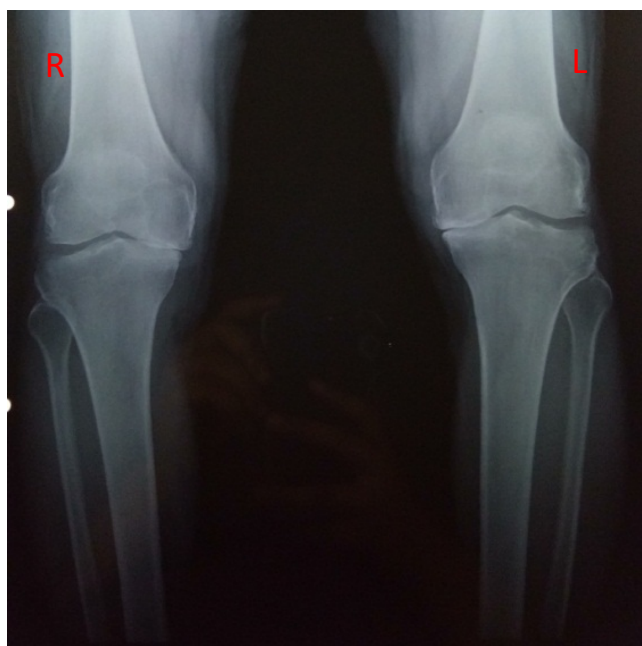

Rt.

FTA $-184^{0}$

JCA $-4^{0}$

Pre-Operative

Radiograph

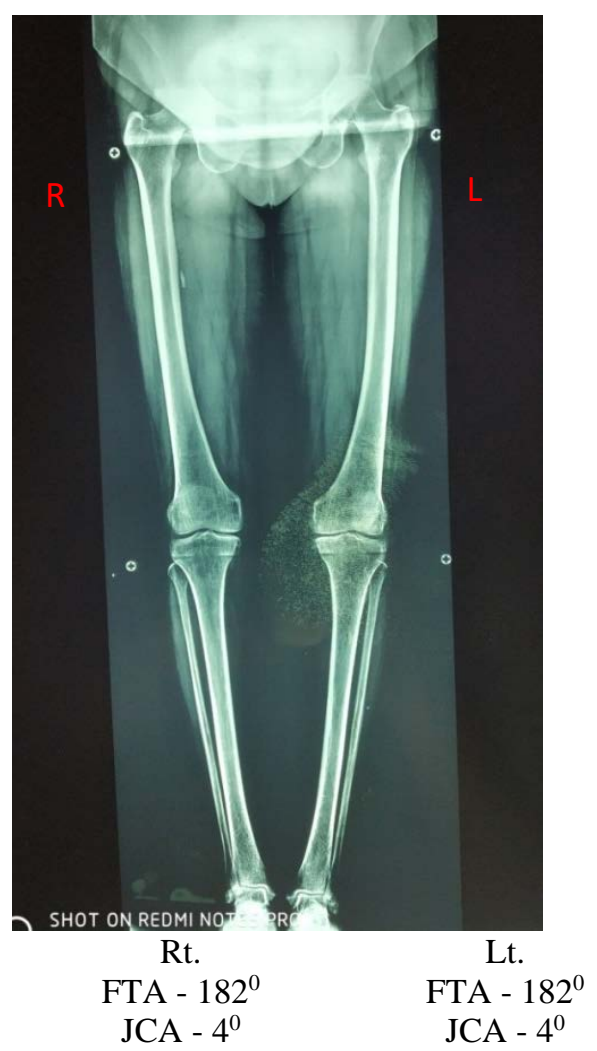

The patients were ambulated as early as possible after the anaesthesia wore off. Also static quadriceps exercise was started and the patients were made to walk as per comfort level. The patients were discharged on day 3 post op.

\section{Results}

- We compared the pre-operative VAS score with the scores at consecutive follow up and at end of three years.

- There was significant improvement in VAS score at each step. At final follow up (3 years) there was significant improvement in VAS from $8.55+0.02$ pre operatively to

\section{Post-Operative}

Radiograph

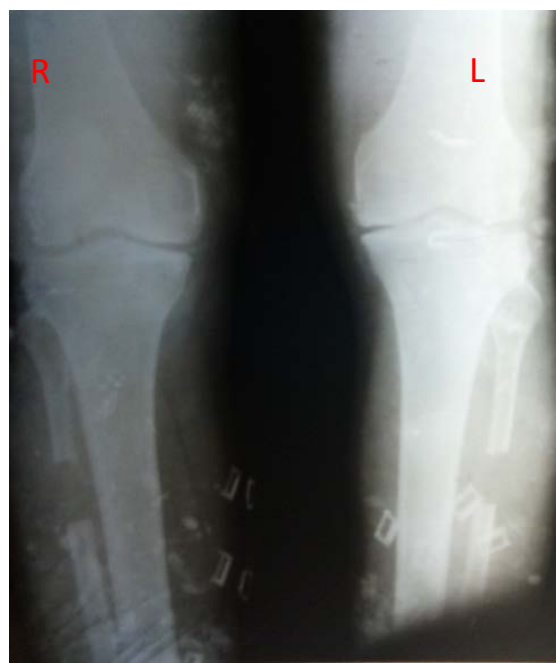

Rt.

Lt.

FTA $-182^{0}$

JCA - $1^{0}$

FTA $-181^{0}$

JCA - $2^{0}$

\section{Post-Operative \\ Radiograph}

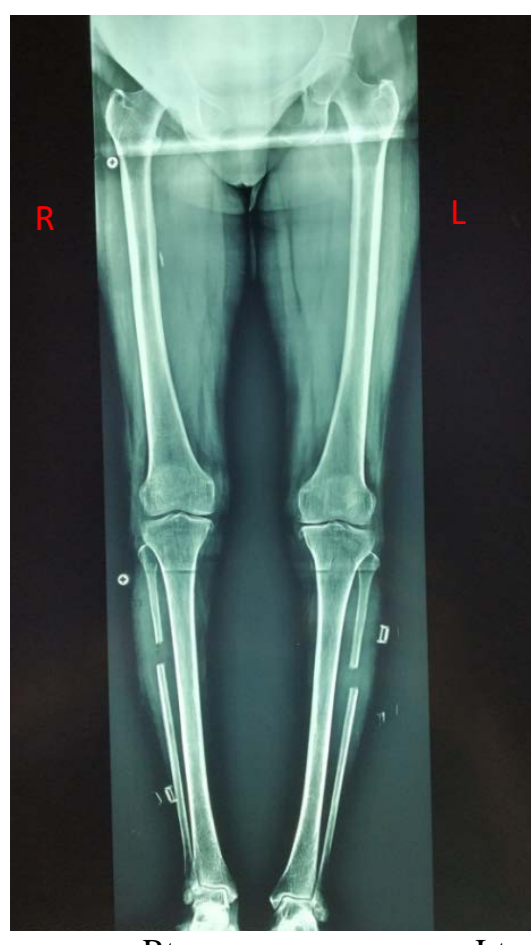

Rt.

Lt.

FTA - $180^{\circ}$

JCA - $2^{0}$

FTA $-180^{\circ}$

JCA - $2^{0}$

$1.87+1.261$

- We compared the pre-operative KSS score with the scores at consecutive follow up. There was significant improvement in KSS score at each step. At final follow up (3 years) there was significant improvement in KSS from $33.55+1.085$ to $85.95+12.989$.

- We compared the pre-operative mean femorotibial angle with the postoperative mean femorotibial angle. There was significant improvement in femorotibial angle from 184.05+1.694 degrees to 181.8+1.159 degrees.

- We compared the pre-operative joint convergence angle 
with the postoperative joint convergence angle. There was significant improvement in joint convergence angle from $3.13+1.223$ degrees to $1.3+0.853$ degrees (On all these values, chi square test was applied and $\mathrm{P}<0.0001$ which is $<0.05$ so statistically significant).

- Patients who regularly took physiotherapy and also did knee strengthening exercise specially quadruceps strengthening, side leg raise remain pain free.

- Patients with normal weight (BMI), were benefitted more.

- 2 patients were lost during follow up.

\section{Complications}

- At final follow up 2 patients were lost in follow up due to death.

- $\quad$ One patient with high BMI and sedentary lifestyle landed up in bilateral total knee replacement

- One female patient with bilateral PFO was pain free for initial two years then she again developed pain in both knee joints on radiograph she was found to have landed up in tricompartmental osteoarthritis.

- During initial first year in our study we observed that there were 3 cases with superficial peroneal nerve injury which recovered within 3-6 months.

- There was one case with swelling over ankle after walking which relieved on taking rest initially and completely resolved after 3 months of surgery.

- $\quad$ One case reported worsening of pain after 6 weeks of surgery which improved after physiotherapy and completely resolved by 12 months.

\section{Discussion}

In our study there were no postoperative complications like DVT, infections etc. Patients were mobilised as early as the anaesthesia effect is weaned off, showed there was significant improvement in medial joint line pain complaints.

There was improvement in American Knee Society Score and VAS score on immediate postoperative day, which gradually improved on consecutive follow up. Patients who were not fatty built and regularly did knee strengthening exercises were benefitted more than with patients with sedentary life style and high BMI.

\section{Conclusion}

In our study after final follow up we conclude that PFO is a novel surgery for medial compartment osteoarthritis of knee with better functional outcome of patients specially in patients with normal to borderline high BMI and active lifestyle and it significant decrease in pain. This surgery reduces the dependence on NSAIDS and delays other costly surgical procedures like high tibial osteotomy and total or unicompartmental knee arthroplasty which require high expertise and carries a large number of complications. Also post operative ambulation is early.

In a developing country like India it can be a promising surgery as it is affordable, minimally associated with complications, doesn't require a high expertise and major surgical procedures like HTO, TKA can be done after this surgery if needed.

Although this surgery carries a lot more advantages, we still need more duration of follow up to conclude how it fares in the long term.

\section{References}

1. Silman AJ, Hochberg MC. 2nd ed. Oxford: Oxford
University Press; Epidemiology of the Rheumatic Diseases 2001.

2. Symmons D, Mathers C, Pfleger B. Global Burden of Osteoarthritis in year: Global burden of disease 2000 study, World health report 2002, (5). Version 2.

3. Akinpelu AO, Alonge TO, Adekanla BA, Odole AC. Prevalence and pattern of symptomatic knee osteoarthritis in Nigeria: A community-based study. Internet J Allied Health Sci Pract 2009;7:3.

4. Zhang YZ. Innovation in Orthopedics and Traumatology in China. Chin. Med J (Engl) 2015;128:2841-2842. 